\title{
New Types of Thermodynamics from $(1+1)$-Dimensional Black Holes
}

\author{
P.T. Landsberg \\ Dept. of Mathematical Sciences \\ University of Southampton \\ Southampton United Kingdom SO9 5NH \\ and \\ R.B. Mann \\ Department of Physics \\ University of Waterloo \\ Waterloo, Ontario \\ N2L 3G1 \\ July 6, 1993 \\ WATPHYS TH-93/05
}




\begin{abstract}
For normal thermodynamic systems superadditivity $\mathbf{S}$, homogeneity $\mathbf{H}$ and concavity $\mathbf{C}$ of the entropy hold, whereas for $(3+1)$-dimensional black holes the latter two properties are violated. We show that $(1+1)$-dimensional black holes exhibit qualitatively new types of thermodynamic behaviour, discussed here for the first time, in which $\mathbf{C}$ always holds, $\mathbf{H}$ is always violated and $\mathbf{S}$ may or may not be violated, depending of the magnitude of the black hole mass. Hence it is now seen that neither superadditivity nor concavity encapsulate the meaning of the second law in all situations.
\end{abstract}


Lower dimensional gravity continues to remain a subject of active research, primarily due to the insights it might yield into the nature of quantum gravity. In particular, although the Einstein tensor is trivially zero in two spacetime dimensions, it has been shown that black holes do exist in this setting [1, []. The associated theory of gravitation from which they arise is formed by setting the Ricci scalar equal to the trace of the conserved stress energy tensor [3]; despite its simplicity, this theory also has a number of other striking classical and semi-classical features, including a well-defined Newtonian limit [3], a post-Newtonian expansion, FRW cosmologies, gravitational collapse [4] and black hole radiation [5, 6, 7]. Indeed, in a certain sense the field equations are the $D \rightarrow 2$ limit of the $D$-dimensional Einstein equations [8]. More recently, it has been shown that $(1+1)$-dimensional black holes can also arise from string-motivated dilaton theories of gravity in two dimensions [9].

Static black hole metrics in two spacetime dimensions are conveniently exhibited in the form

$$
d s^{2}=-\alpha(x) d t^{2}+\alpha^{-1}(x) d x^{2}
$$

where $\alpha(x)$ is determined from field equations which follow from an action for two dimensional gravity whose general form we shall outline below. The spacetime (1) is that of a static $(1+1)$ dimensional black hole if $\alpha(x)<0$ for some spatial region $x \in(b, a)$. Dimensional arguments [5] indicate that the temperature associated with such black holes is proportional to the ADMmass M. This quantity has units of inverse length, and so metrics of the form (11) depend upon $M$ as $\alpha=\alpha\left(M x, Q_{p} / M^{p}\right)$, where $Q_{p}$ is a coupling constant that appears in the matter action of dimension (length) ${ }^{-p}$. Standard Wickrotation arguments [1] yield

$$
T=\frac{M}{2 \pi}\left|\frac{d \alpha\left(y, Q_{p} / M^{p}\right)}{d y}\right|_{y_{H}}
$$

where the horizon $x_{H}=y_{H}(M) / M$ is defined via $\alpha\left(y_{H}, Q_{p} / M^{p}\right)=0$. Provided that $\frac{d \alpha\left(y, Q_{p} / M^{p}\right)}{d y}$ is independent of $Q$ (a situation which holds in a large number of cases [5, 10]) the entropy varies as $\ln \left(M / M_{0}\right)$, where $M_{0} \neq 0$ is a constant of integration which arises from integrating the thermodynamic relation $d U=T d S$. As the black hole radiates away its mass its Compton wavelength becomes comparable to its Schwarzchild radius, beyond which 
the thermodynamic limit breaks down and a full quantum theory of gravity is required. The zero of entropy may be chosen to be at this mass scale, thereby defining the constant $M_{0}$. We shall assume henceforth that this situation holds.

The $(1+1)$-dimensional black hole gives rise to some interesting points of principle in thermodynamics as will be shown in this paper. In order to explain this we recall some relevant earlier results [11, 12, 13]. Let $X_{a}, X_{b}$ be two values of a set of extensive variables referring to a specific system like a gas, a solid, a black hole, etc. Then the entropy can satisfy any of the following properties:

$$
\begin{aligned}
\text { superadditivity } & \mathbf{S}: & S\left(X_{a}+X_{b}\right) & \geq S\left(X_{a}\right)+S\left(X_{B}\right) \\
\text { homogeneity } & \mathbf{H}: & S\left(\mu X_{a}\right) & =\mu S\left(X_{a}\right) \\
\text { concavity } & \text { C : } & S\left(\lambda X_{a}+(1-\lambda) X_{b}\right) & \geq \lambda S\left(X_{a}\right)+(1-\lambda) S\left(X_{b}\right)
\end{aligned}
$$

where $\mu$ and $\lambda(0 \leq \lambda \leq 1)$ are arbitrary positive constants.

$\mathbf{S}$ states that on withdrawing a partition so that two systems of a similar type can merge, the entropy increases or remains unchanged. $\mathbf{H}$ asserts the extensive nature of the entropy and $\mathbf{C}$ is its concavity. Note that

$$
\mathrm{S}+\mathrm{H} \Rightarrow \mathrm{C}
$$

which holds because $\mathbf{S}$ and $\mathbf{H}$ respectively imply $S(\lambda X+(1-\lambda) Y) \geq S(\lambda X)+$ $S((1-\lambda) Y)=\lambda S(X)+(1-\lambda) S(Y)$, which is C. Similarly,

$$
\mathrm{C}+\mathrm{H} \Rightarrow \mathrm{S}
$$

because $\mathbf{C}$ and $\mathbf{H}$ respectively imply $S(\lambda X+(1-\lambda) Y) \geq \lambda S(X)+(1-$ $\lambda) S(Y)=S(\lambda X)+S((1-\lambda) Y)$; setting $\lambda X \equiv X_{a}$ and $(1-\lambda) Y \equiv X_{b}$ yields S .

As a consequence, of the eight conceivable types of thermodynamics

$$
\begin{array}{llll}
\text { SHC } & \text { SHC } & \underline{S H C} & S \bar{H} \bar{C} \\
\overline{\bar{S} H C} & \overline{\mathrm{S}} \bar{H} C & \overline{\mathrm{S} H \bar{C}} & \overline{\mathrm{S}} \bar{H} \bar{C}
\end{array}
$$

not all are logically admissible; we have underlined the inadmissible ones in (6) above. A third implication

$$
\mathrm{S}+\mathrm{C} \Rightarrow \mathrm{H}
$$


may be proved subject to the assumption that $\mathbf{S}(0)=0$ [14]. If (77) holds, this would imply that

\section{$\mathrm{S} \overline{\mathrm{H} C}$}

is not allowed.

Up to the present it was believed that $\mathbf{S}$ was a requirement, leaving only the three types in the top line of (6), and only two if (8) is excluded. Of these, SHC is indeed the normal type of thermodynamics, while $\mathbf{S} \overline{\mathbf{H}} \overline{\mathbf{C}}$ applies to three-dimensional black holes of mass $M$ for which the entropy $S=\frac{4 \pi G}{k} M^{2}$ ensures that $\mathbf{H}$ and $\mathbf{C}$ are violated. This then provided examples of apparently all available types of thermodynamics.

For $(1+1)$-dimensional black holes, we will show that the dimensionless entropy

$$
\sigma_{0} \equiv \frac{2 \pi}{\hbar k} S=\ln \left(\frac{M}{M_{0}}\right)
$$

satisfies $\mathbf{S H} \mathbf{C}$ for $M / M_{0}<4$ and $\overline{\mathbf{S}} \overline{\mathbf{H}} \mathbf{C}$ for $M / M_{0}>4$, thus adding an example for each of two relatively unexplored types of thermodynamics. It is the circumstance that $S(0) \neq 0$ that brings the type (8) into play. The fact that $S(0)$ is negative is a key characteristic of the MATHEMATICAL equation for $S(X)$. Note that therefore its PHYSICAL applicability must cease just before the dashed portions of the curves of fig. 1 are reached.

Consider a $(1+1)$-dimensional system consisting of a black hole of mass $M$ which has spawned $n$ identical black holes of mass $m$. The dimensionless entropy is

$$
\sigma_{n}=\ln \left[\frac{M-n m}{M_{0}}\left(\frac{m}{M_{0}}\right)^{n}\right]
$$

provided the subsystems are sufficently separated to be regarded as independent. Eq. (10) becomes (9) for $n=0$. It is easily confirmed that for a given $n, M$ and $M_{0}, \sigma_{n}$ has its largest value if $m=M /(n+1)$, whence

$$
\sigma_{n}^{\max }=(n+1) \ln \left[\frac{M}{(n+1) M_{0}}\right] .
$$

Fig. 1 shows a plot of $\sigma_{1}$ as a function of $m / M_{0}$ and also gives an indication of $\sigma_{0}$. The changes indicated by arrows in fig.1 must occur because the second law states, for our purposes here and for many other purposes as well, that the entropy increases, or possibly stays constant, in spontaneous processes. 
For $M / M_{0}=3$ the separated black holes merge, and the system is of the type SHCF; for $M / M_{0}=5$ the black hole tends to split into equal parts and the system is of the type $\overline{\mathbf{S}} \overline{\mathbf{H}} \mathbf{C}$. The concave nature of the logarithmic function ensures the validity of $\mathbf{C}$ in both cases.

For $n$ large enough to permit differentiation of $\sigma_{n}^{\max }$ with respect to $n$, one finds (given $M$ and $M_{0}$ ) the maximal value of $\sigma_{n}^{\max }$ (say, $\hat{\sigma}_{n_{0}}$ ) to be at $n=n_{0}$, where

$$
n_{0}=\frac{M}{e M_{0}}-1 \quad \text { and } \quad \hat{\sigma}_{n_{0}}=\frac{M}{e M_{0}}=n_{0}+1 .
$$

Strictly speaking $n_{0}$ must be an integer and $\hat{\sigma}_{n_{0}}$ is a rising staircase curve as a function of $n_{0}$.

To find the entropically best transition for a black hole of mass $\mathrm{M}$ one must ensure that $\sigma_{n}>\sigma_{0}$, or alternatively $M / M_{0}>(n+1)^{1+1 / n}$. This yields the number of equal black holes into which the original one will fragment. Equating $\sigma_{0}$ and $\sigma_{1}$ yields a curve of "indifference" when the black hole system can split up, or not, without a change of entropy. This occurs for

$$
\frac{M}{M_{0}}=4 \quad \text { where } \quad \sigma_{0}=\sigma_{1}=2 \ln (2)=1.386 \text {. }
$$

This case is also illustrated in Fig. 1. For no fragmentation $M / M_{0}$ is less than 4; so the fragmentation process always comes to a stop, as one intuitively expects.

The entropy formula (9) has been shown to follow from the static black hole metric (1) using both naive Wick rotation and more rigorous quantum field theoretic arguments [5]. Such metrics arise from theories of $(1+1)$ dimensional gravity based on the action

$$
S[g, \Psi]=\int d^{2} x \sqrt{-g}\left(H(\Psi) g^{\mu \nu} \nabla_{\mu} \Psi \nabla_{\nu} \Psi+D(\Psi) R+V\left(\Psi ; \Phi_{M}\right)\right)
$$

where $R$ is the Ricci scalar and $D$ and $H$ are arbitrary functions of the scalar field $\Psi$ (referred to as the dilaton in string theory). The potential $V$ is the matter Lagrangian, and depends on both $\Psi$ and the matter fields $\Phi_{M}$; in general the associated conserved stress-energy tensor $T_{\mu \nu}$ will depend upon both fields. One need only require that static metrics which are solutions to the field equations which follow from (14) have the asymptotic form

$$
\alpha(x) \rightarrow 2 M|x|-1
$$


for large $|x|$ since in this case the metric (四) will become asymptotically like a Rindler spacetime; a Rindler transformation may then be applied locally to rewrite the metric in Minkowskian form. Collapse of $(1+1)$ dimensional dust [4 has been shown to yield black hole metrics whose exterior metric matches onto solutions with such asymptotic behaviour.

As an example of a specific black hole metric, if $D(\Psi)=\Psi, H=1 / 2$ and $V(\Phi)$ is the the stress-energy tensor of a Liouville field coupled to gravity, then

$$
\alpha(x)=1-q e^{-2 M|x|}
$$

where $\Lambda=q M^{2}$ is the coupling constant. The parameter $M$ is the ADMmass [10 and we have set $2 \pi G=1$. Note that $(\sqrt{16})$ is also the black-hole metric of string theory [9] if one interchanges the parameters $M$ and $\sqrt{\Lambda}$; this yields radically different thermodynamics from that which we are considering. The metric (16) is asymptotically flat for large $|x|$, has an event horizon at $|x|=\ln (q) /(2 M)$, and a temperature $M /(2 \pi)$. Our discussion here is also pertinent to the case of $(2+1)$ dimensional black holes [15], for which $T \sim \sqrt{M}$ and $S \sim \sqrt{M}$, again leading to a breakdown of superadditivity.

We should explain that one really needs the dynamics of black holes moving apart in order to explain how they can fragment. This is presumably a hard GR problem. All one can be sure about is that the final state has a higher entropy.

Can the essence of the second law of thermodynamics be conveyed by a functional property of the entropy (in terms of key extensive variables like energy, volume, mass etc.)? It has been maintained by some that it resides in the superadditivity of the entropy and by others that it resides in the concavity of the entropy. We have shown here that neither position can now be maintained universally. $\mathbf{H}$ and $\overline{\mathbf{H}}, \mathbf{C}$ and $\overline{\mathbf{C}}$ and also $\mathbf{S}$ and $\overline{\mathbf{S}}$ can characterize the entropy. Our study of $(1+1)$-dimensional black hole thermodynamics has doubled the number of useful and relevant types of thermodynamics from two to four. This still leaves one with a search for physically relevant examples for the types $\overline{\mathbf{S}} \mathbf{H} \overline{\mathbf{C}}$ and $\overline{\mathbf{S}} \overline{\mathbf{H}} \overline{\mathbf{C}}$.

\section{Acknowledgements}

In ref. [13], tables 5 and 6 , we put $\overline{\mathbf{S}}, \overline{\mathbf{H}} \Rightarrow \mathbf{C}$, when only $\overline{\mathbf{S}}, \overline{\mathbf{H}}$ should have been written, nor was the assumption $S(0)=0$ explicitly noted in 
[13], 14] for $\mathbf{S}+\mathbf{C} \Rightarrow \mathbf{H}$. We are indebted to Dr. R. Woodard, University of Florida, Gainesville for enlightening discussions of the various types of thermodynamics. This work was supported in part by the Natural Sciences and Engineering Research Council of Canada.

\section{References}

[1] R.B. Mann, A. Shiekh, and L. Tarasov, Nucl. Phys. B341 (1990) 134.

[2] J.D. Brown, M. Henneaux and C. Teitelboim, Phys. Rev. D33 (1986) 319; J.D. Brown, Lower Dimensional Gravity, (World Scientific, 1988).

[3] R.B. Mann, Found. Phys. Lett. 4 (1991) 425.

[4] A.E. Sikkema and R.B. Mann, Class. Quantum Grav. 8 (1991) 219 .

[5] R.B. Mann and T.G. Steele, Class. Quant. Grav. 9 (1992) 475;

R.B. Mann, L. Tarasov and A. Zelnikov, Class. Quant. Grav. 9 (1992) 1487.

[6] R.B. Mann, S.M. Morsink, A.E. Sikkema and T.G. Steele, Phys. Rev. D43 (1991) 3948.

[7] S.M. Morsink and R.B. Mann, Class. Quant. Grav. 8 (1991) 2257.

[8] R.B. Mann and S.F. Ross, Class. Quant. Grav. (to be published).

[9] G. Mandal, A.M. Sengupta, and S.R. Wadia, Mod. Phys. Lett. 6 (1991) 1685.

[10] R.B. Mann, Phys. Rev. D47 (1993) 4438.

[11] P.T. Landsberg and D. Tranah, Collective Phenomena 3 (1980) 73. 
[12] D. Tranah and P.T. Landsberg, Collective Phenomena 3 (1980) 81.

[13] P.T. Landsberg in Black Hole Physics, eds. V. de Sabbata and Z. Zhang, (Dordrecht, Kluver 1992).

[14] P.T. Landsberg and D. Tranah, Phys. Lett. A78 (1980) 219.

[15] M. Bañados, C. Teitelboim and J. Zanelli, Phys. Rev. Lett. 69, 1849 (1992); R.B. Mann and S.F. Ross, Phys. Rev. D47 (1993) 3319. 


\section{Figure Captions}

Fig. 1 The dimensionless entropy of a combination of two separated $(1+1)$ dimensional black holes of masses $M-m$ and $m$ as a function of $m / M_{0}$. For $M / M_{0}=3$ on has the $\mathbf{S} \overline{\mathbf{H}} \mathbf{C}$ type of thermodynamics and for $M / M_{0}=5$ one has the $\overline{\mathbf{S}} \mathbf{H} \mathbf{C}$ type. The curve $M / M_{0}=4$ represents a limiting case. 